\title{
COLABORACIÓN ACTIVA EN WIKIPEDIA COMO MÉTODO DE APRENDIZAJE
}

\author{
(USING ACTIVE COLLABORATION IN WIKIPEDIA AS A LEARNING TOOL)
}

$\mathrm{M}^{\mathrm{a}}$ Isabel Alonso de Magdaleno

Jesús García García

Universidad de Oviedo (España)

\section{RESUMEN}

Las actuales generaciones de alumnos han crecido en un entorno donde las principales fuentes de información se encuentran en formato digital. Por ello, en el ámbito de los trabajos escritos, los autores proponemos la edición de entradas de Wikipedia como herramienta de un proceso constructivo y de razonamiento crítico que mejore los conocimientos adquiridos, además de perfeccionar las habilidades de manejo, citación de fuentes y presentación de información. Previamente, se constata mediante un estudio empírico el comportamiento del alumnado en la realización de trabajos escritos. Este comportamiento resulta coherente con lo expresado en la literatura previa.

Palabras clave: conocimiento abierto, trabajos escritos, wikipedia, calidad, colaboración.

\begin{abstract}
Our current generation of students has grown up in an environment where the main source of information is now in digital format. For this reason, the authors propose, within the context of written assignments, the editing of Wikipedia entries as a tool for constructive and critical thinking. In this way, they can improve the following areas: the acquisition of knowledge, information management skills, and develop proper citation skills, as well as improve other skills related to information management. Previously, however, this was done by means of an empirical study of the students' behavior in relation to written work. This behaviour proved to be consistent with previous literature about the subject.
\end{abstract}

Keywords: open knowledge, written assignments, wikipedia, quality, collaboration. 
Una de las herramientas más empleadas del proceso de enseñanza-aprendizaje es la asignación de trabajos escritos, especialmente en los procesos de educación a distancia. Con frecuencia, el profesor encuentra que los trabajos carecen de suficiente reflexión crítica por parte del alumnado, limitándose a lo que coloquialmente se denomina un copia y pega de unas pocas fuentes obtenidas de Internet. Como resultado, se obtienen unos trabajos que se limitan a plasmar una información básica que se puede extraer con sencillez de Internet.

A través de un estudio empírico hemos querido contrastar el comportamiento de los alumnos en la realización de trabajos escritos. Los datos obtenidos nos permiten corroborar en la práctica las hipótesis antes mencionadas. Como consecuencia, creemos que el perfeccionamiento del proceso de enseñanza-aprendizaje ha de aprovechar lo que ya es prácticamente un hecho cultural. Internet es el primer recurso empleado en la búsqueda de información y tanto aprendizaje como evaluación no pueden permanecer ajenos a este hecho.

\section{CONOCIMIENTO ABIERTO}

El concepto de conocimiento abierto, referido en ocasiones como conocimiento libre, no debe ser confundido con el concepto de acceso abierto. El primero deriva del concepto de software libre (Free Software Foundation, 1986) y se basa en una serie de premisas acerca de qué se puede hacer con el conocimiento. El segundo se refiere fundamentalmente al acceso inmediato y sin restricciones (registro, suscripción o pago) a material digital educativo, académico, científico o de cualquier otro tipo, y se ha centrado principalmente en el acceso a artículos de investigación científica de revistas especializadas (Budapest Open Access Initiative, 2001; Open Knowledge Foundation, 2004).

Sin una definición universalmente aceptada, podemos calificar como conocimiento libre a aquel que reúne una serie de características, que podríamos resumir como (Declaración de Berlín sobre Acceso Abierto, 2003):

- Acceso íntegro a un coste de reproducción razonable o gratuitamente en Internet.

- Redistribución gratuita y sin limitaciones de ningún tipo.

- Reutilización libre para modificaciones y obras derivadas, admitiéndose las restricciones de reconocimiento de autoría original y/o integridad del contenido si el autor original así lo decidiera. 
- Ausencia de restricciones tecnológicas que limiten acceso, reutilización o redistribución; preferiblemente la obra debe plasmarse en formatos digitales libres y estándares.

Wikipedia se amolda, en todas sus versiones, a esta definición de conocimiento libre, conceptualizándose como una enciclopedia en línea y de carácter políglota con más de 20 millones de artículos en 282 idiomas y dialectos. Es la quinta Web más visitada del mundo y la mayor obra de referencia existente en la Red. Su contenido se encuentra disponible libremente bajo licencias GNU y Creative Commons Attribution Share-Alike, lo que impone las condiciones de atribución de autoría y mantenimiento de la misma licencia a los contenidos redistribuidos pero no impide la atribución de los derechos de autor según la Convención de Berna a cada editor o ilustrador que realice aportaciones (Wikipedia, 2009).

En consecuencia, creemos que Wikipedia constituye no solo un recurso educativo por sus contenidos sino también una poderosa herramienta de aprendizaje. En el siguiente apartado, expondremos las razones que nos llevan a sostener esta afirmación; afirmación que es consistente con la iniciativa Wikipedia Global University Program impulsada por Wikimedia Foundation, organización operadora del proyecto enciclopédico (Wikipedia, 2011).

\section{PAUTAS DE COMPORTAMIENTO EN LA REALIZACIÓN DE TRABAJOS ESCRITOS}

\section{Fundamentos previos}

La copia textual o con ligeras modificaciones sin acreditar la fuente de procedencia siempre ha estado presente en la realización de los trabajos escritos. Recientemente, un estudio realizado sobre estudiantes de secundaria en Finlandia mostraba que la Web era casi exclusivamente la única fuente de información empleada para la realización de trabajos escritos y que dichas fuentes no eran acreditadas en un $30 \%$ de los casos; además, un tercio de los contenidos eran copia literal o muy ligeramente transformadas de otras fuentes, fueran citadas o no citadas (Sormunen y Lehtiö, 2011).

La explicación a este tipo de comportamientos ha sido ya expuesta previamente: los estudiantes, en especial los de menor perfil académico, no se encuentran excesivamente interesados en investigar y aprender sobre la cuestión en estudio sino que perciben los trabajos escritos como ejercicios de búsqueda en los que primaría 
la localización de respuestas correctas que serían transferidas al trabajo escrito (Alexandersson y Limberg, 2003). Esta hipótesis, enfocada en la primacía de las habilidades de búsqueda sobre la reflexión crítica en el contenido de las fuentes, fue confirmada por estudios posteriores del mismo ámbito escolar nórdico (Heinström, 2006; Limberg et al., 2008).

Respecto a las fuentes empleadas y citadas en las referencias de los trabajos, los mismos estudios parecían determinar que en muchas ocasiones, dichas fuentes cuando existen, son añadidas con la finalidad de cumplir con los requisitos académicos, no siendo correctamente interpretadas ni aplicadas al problema en estudio. Este hecho puede ser en parte explicado porque los alumnos no han sido previamente instruidos por el profesor en la importancia de la atribución de citas $\mathrm{y}$, por tanto, son percibidas como una complejidad añadida que no contribuye al proceso de búsqueda de respuestas correctas (Forte y Bruckman, 2010).

\section{Diseño de la investigación}

Para poder confirmar y cuantificar de manera empírica las rutinas de los estudiantes en la realización de trabajos escritos, se ha aplicado una encuesta entre alumnos de diversas titulaciones de la Universidad de Oviedo. La muestra total se compuso de 86 alumnos de las titulaciones de Ingeniería Industrial (primer curso) e Ingeniería Química (quinto curso) que respondieron a un cuestionario anónimo en el cual se les solicitaba que indicaran el orden de preferencia en el uso de diversas fuentes para la búsqueda de información y que mostraran su grado de acuerdo o desacuerdo con una serie de proposiciones referentes al uso de las fuentes y también respecto a su grado de familiaridad con los conceptos de conocimiento y software libre. La formulación de las fuentes, del uso de Internet y los enunciados declarativos, se basaron en la experiencia docente de los autores.

\section{Resultados}

En los resultados obtenidos respecto a las fuentes empleadas en los trabajos escritos (figura 1), no resulta sorprendente que la primera fuente empleada sea Internet, seguida a gran distancia de consultas en bibliotecas, consulta de libros propios o consultas a otras personas que son prácticamente residuales. Que la Red sea la primera fuente para prácticamente todos los alumnos, la convierte en el campo ideal para llevar a cabo cualquier innovación educativa respecto a la realización de trabajos y no hace sino reafirmar su valor como vector de conocimiento. 


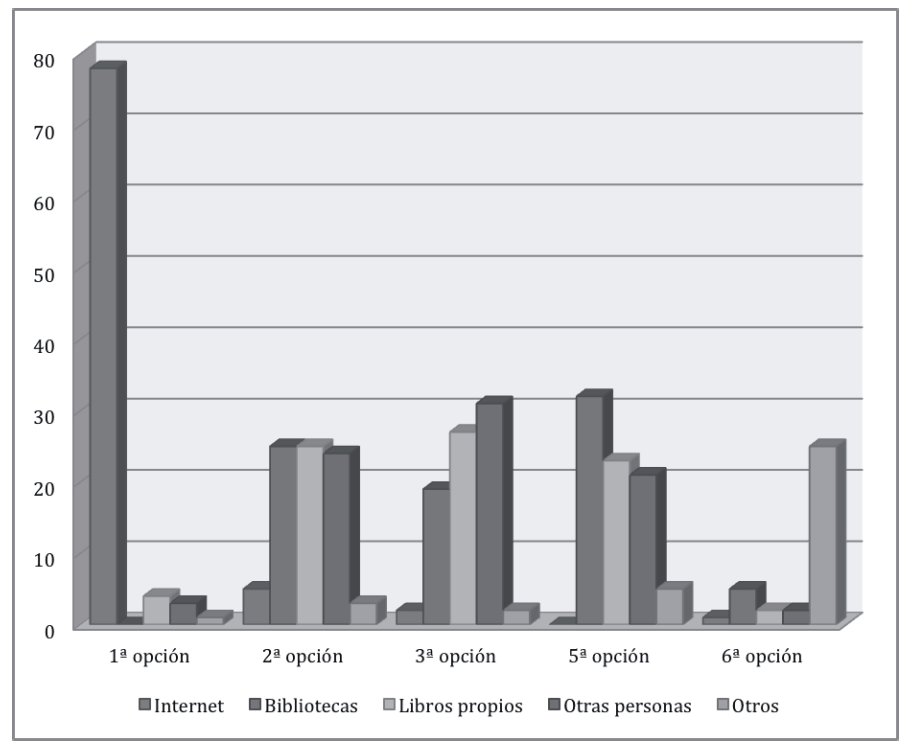

Figura 1. Priorización de fuentes empleadas

En este amplio uso de la Red, el recurso más empleado es el buscador Google, utilizando como fuentes los primeros resultados ofrecidos en la búsqueda y, en muchas ocasiones, solo el primer resultado; seguido por la búsqueda directa en la enciclopedia libre en línea Wikipedia. Estas tres opciones constituyen los dos primeros recursos empleados por la casi totalidad de los alumnos encuestados, como se puede observar en los resultados mostrados en la figura 2. Este comportamiento nos inclina aún más a afirmar que cualquier acción de mejora de la calidad en los trabajos escritos debe realizarse en torno a estos recursos para gozar de una mayor aceptación. 


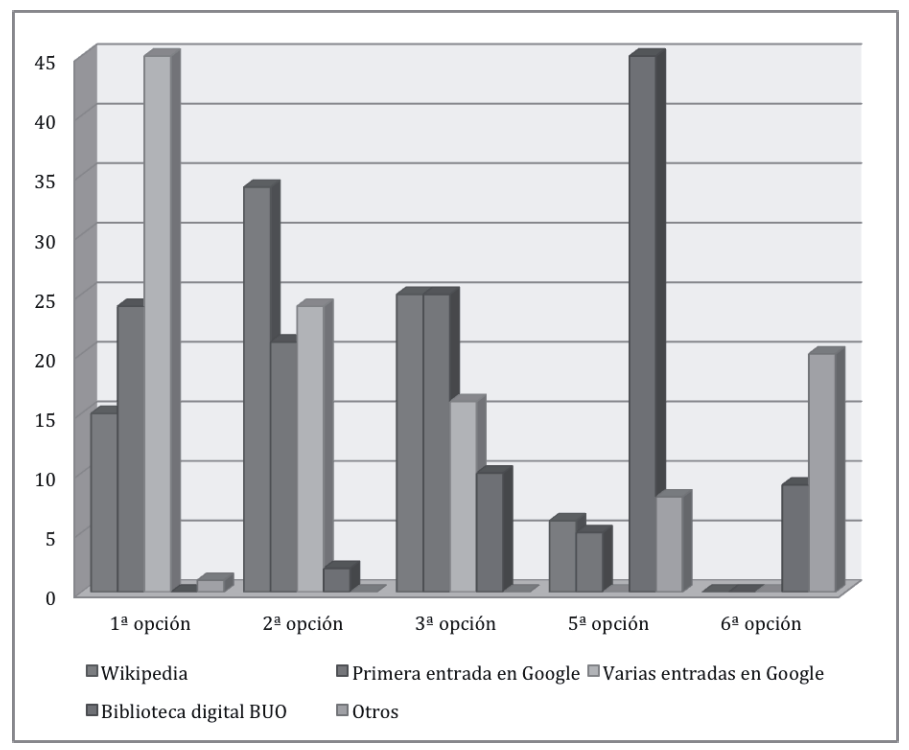

Figura 2. Priorización de recursos digitales empleados

Dentro del uso de recursos digitales destaca el escaso uso que se realiza de la biblioteca digital de la Universidad de Oviedo. Esta plataforma cuenta con más de 70.000 libros electrónicos en diversas lenguas y de las más variadas materias, más de 42.00o títulos de revistas científicas y más de un centenar de bases de datos. No obstante, no resultando desconocida para los estudiantes, no es empleada por estos en sus tareas académicas. Entre las razones que suponemos detrás de este escaso uso se encontrarían la sencillez de manejo de las alternativas de búsqueda de información a través de Google y las limitaciones a la exportación de información en la mayor parte de las publicaciones, normalmente PDFs u otros formatos leídos a través de aplicaciones privativas que limitan el proceso de copia y pega. Es natural imaginar que los estudiantes tratan la información en formatos que faciliten su manejo; en este sentido, las bibliotecas digitales basadas en formatos privativos solo establecen barreras a este tratamiento por lo que no ha de extrañar su baja tasa de uso.

Una vez establecida la priorización de fuentes, los siguientes apartados del cuestionario trataron de que los estudiantes manifestaran su acuerdo o desacuerdo con ciertos comportamientos fijados en declaraciones que se proponían para su valoración y que ofrecemos en la tabla 1. Los resultados se muestran en la figura 3. 


\begin{tabular}{|l|l|}
\hline Declaración 1 & Al realizar mis trabajos copio y pego resultados de una única fuente. \\
\hline Declaración 2 & $\mathrm{Al}$ realizar mis trabajos copio y pego de varias fuentes. \\
\hline Declaración 3 & $\begin{array}{l}\mathrm{Al} \text { realizar mis trabajos examino varias fuentes y elaboro una redacción } \\
\text { propia. }\end{array}$ \\
\hline Declaración 4 & $\begin{array}{l}\text { En mis trabajos cito la totalidad de las fuentes de las que obtuve } \\
\text { información. }\end{array}$ \\
\hline
\end{tabular}

Tabla 1. Declaraciones propuestas para su valoración

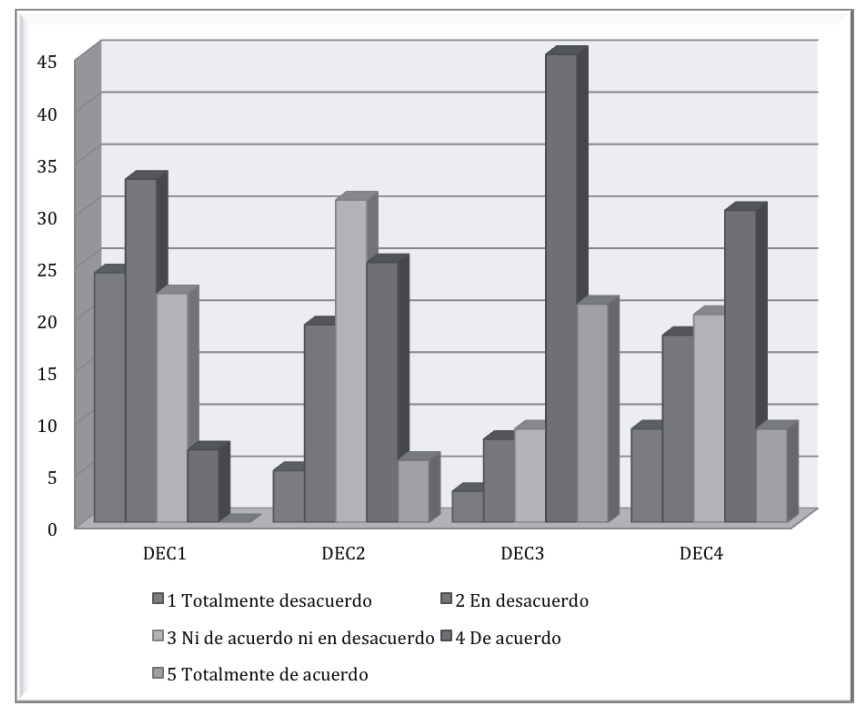

Figura 3. Valoración de declaraciones

Los alumnos manifestaron su desacuerdo mayoritario con la realización de copia y pega de una única fuente. Pero este desacuerdo ya no era mayoritario cuando declaraban que este copia y pega se producía de varias fuentes. No obstante, una amplia mayoría manifestaba realizar una redacción propia de lo obtenido de ellas. Por último, algo menos de la mitad de los alumnos declararon citar las fuentes en que se habían basado, lo que confirma que la atribución de autoría es uno de los principales problemas en los trabajos escritos.

Respecto a la atribución de la fuente sería necesaria una indagación adicional para confirmar si es debida a una falta de cultura sobre la necesidad moral de asignar méritos a los autores de ideas o textos originales o si es debida a un deseo de tratar de enmascarar un trabajo ajeno como propio. 


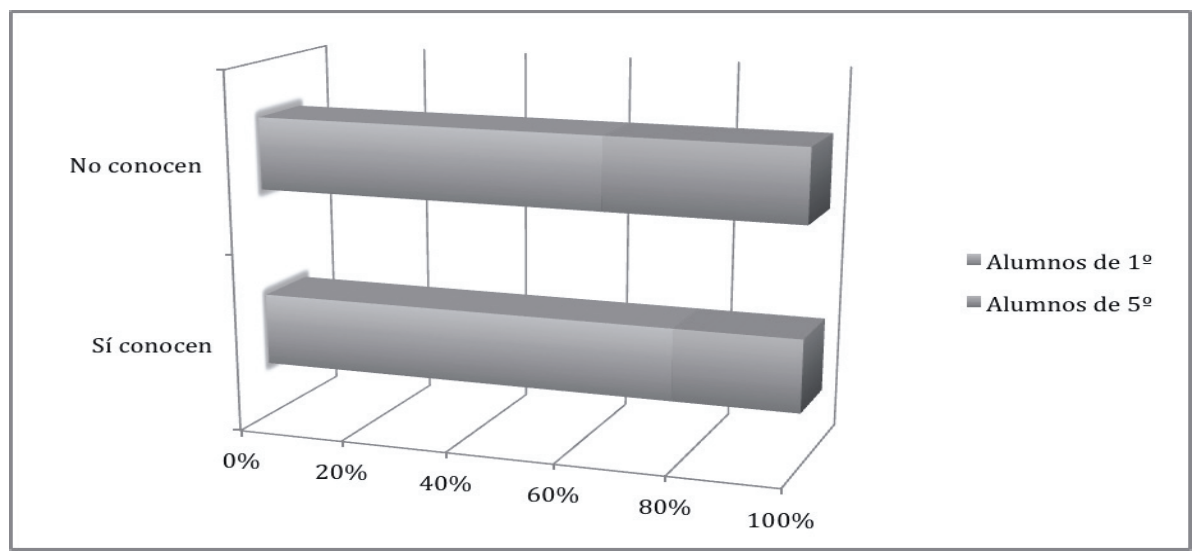

Figura 4. Grado de comprensión del concepto conocimiento libre

Por último, pudimos contrastar que más de la mitad de los alumnos encuestados manifestaban conocer y comprender lo que significa el concepto de conocimiento libre o abierto o, en su defecto, el concepto de software libre basado en los mismos valores. No obstante, una pequeña cifra de ellos confundía la noción de libertad con la de gratuidad o no los diferenciaba de manera suficiente en las preguntas de control, entendiendo por conocimiento y/o software libre aquel que se encontraba disponible para su uso de manera gratuita. Consecuentemente, no captaban como parte integral del concepto la redistribución y, sobre todo, la reutilización de ese conocimiento como base para obras derivadas.

En base a estos resultados, nace la idea de trabajar con la edición de entradas de Wikipedia como herramienta para la mejora de la calidad en los trabajos escritos.

\section{LA EDICIÓN DE WIKIPEDIA COMO HERRAMIENTA DE MEJORA}

Wikipedia constituye una excelente plataforma para la realización de tareas escritas, pues ofrece una serie de ventajas en el trabajo con los estudiantes:

- Se encuentran familiarizados con ella ya que la emplean con frecuencia como fuente de información, incluso en el ámbito universitario (Head y Eisenberg, 2010). Cercanía y gratuidad, familiaridad con su uso y populares comparativas de calidad, como la realizada por la revista Nature (Giles, 2005), la convierten en un recurso ideal. 
- Si bien le conceden criterio de autoridad como fuente de información, son conscientes de que en ocasiones existen problemas de credibilidad en sus contenidos. Estos problemas son crecientes cuanto más reducido es el número de editores y bajo otra serie de circunstancias como, por ejemplo, artículos de actualidad, polémicas de carácter político o social, etc. (Gourdain et al., 2008).

- Son conscientes de que lo que reflejen en ella se verá sometido a exposición pública, incluyendo a sus propios compañeros, lo que estimula la búsqueda de la excelencia (Schulenburg y Wannemacher, 2009; Konieczny, 2010).

Como hemos expuesto anteriormente, las estrategias de búsqueda y manejo de información empleadas por los estudiantes se basan en la búsqueda de diversas fuentes, primando los contenidos fácilmente accesibles sobre aquellos que les establecen barreras. Por ello, nuestra propuesta consiste en que la realización de trabajos escritos se base en la edición de artículos en Wikipedia, pues se posibilitaría el trabajo en un soporte familiar, en cuyo manejo presentan experiencia, y se generaría un resultado final que permitiría ver su esfuerzo como útil para otros estudiantes, que en el futuro podrían acudir a dicha entrada como fuente de información.

La asignación del trabajo se realizaría tomando como base la información contenida en una entrada prefijada, de manera que la información básica sobre el tema en estudio ya se consideraría como dada, implicando la adquisición de conocimientos adicionales. De esta manera se evitaría la presentación de información básica como contenido principal del trabajo, situando el nivel de esfuerzo deseado más allá de los conocimientos básicos.

En el desarrollo de la asignatura se realizaría una propuesta de trabajo para cada grupo de alumnos, correspondiéndose con una entrada en la Wikipedia que debería ver mejorada sustancialmente su calidad. La metodología de edición de Wikipedia, que permite la participación del editor como usuario registrado y que genera para cada entrada un histórico de cambios, serviría para realizar el control del trabajo de los distintos grupos de alumnos con independencia de los cambios que puedan realizar editores ajenos a la asignatura. Los requisitos de introducción en la edición de información elaborada de manera original y con enlaces a las fuentes que sustenten datos, hechos y afirmaciones introducen al alumnado en la necesidad de citar las fuentes que haya empleado. Que el punto de partida sea una entrada con contenido ya existente, anula dicho contenido básico como integrante del trabajo, permitiendo valorar aportaciones que se encuentren un paso más allá del conocimiento básico sobre la temática. 
En la fecha de presentación del trabajo, los alumnos expondrían sus aportaciones a la entrada en Wikipedia, con especial mención a porqué decidieron qué contenidos eran relevantes para enriquecer el texto preexistente y a la calidad de las fuentes empleadas. Esta presentación ha de ser necesariamente el resultado de un esfuerzo crítico, al basarse el trabajo en la construcción sobre algo ya existente y al estar las modificaciones realizadas expuestas a la revisión de otros editores ajenos a la asignatura que pudieran trabajar sobre la misma entrada. Además, la agregación a un material ya existente, con fuentes disponibles y con unas normas de edición de Wikipedia, que exigen la cita y referencia, motiva la necesidad de atribuir la autoría a las fuentes empleadas.

Participar en la edición de Wikipedia ha de suponer también un aliciente para el estudiante que pasaría a conocer y valorar esta atribución de citas y autorías; se soslayaría la visión del trabajo como un esfuerzo en la localización de respuestas correctas que muestran muchos alumnos para pasar a ser considerado como la realización de una obra a disposición pública y que en el futuro podría ser usada por muchos otros estudiantes, tal y como ellos mismos han usado previamente otra información.

Entre la dificultad de esta operativa de trabajo se podrían encontrar diversas situaciones propias de una obra de la envergadura de Wikipedia. Los distintos trabajos asignados pueden corresponderse con entradas que en el momento de la asignación tengan distintos niveles de contenido. Sería misión del profesor seleccionar entradas cuyo nivel de desarrollo fuera homogéneo, pues en caso contrario un grupo de alumnos podría percibir que su tarea es más difícil que la de otro grupo si estos últimos partieran de una entrada menos desarrollada. Asimismo, los alumnos podrían enfrentarse a la propia cultura de edición de Wikipedia como barrera. En ocasiones, ciertas entradas pueden encontrarse bloqueadas por el editor principal por considerarse polémicas; en otras ocasiones, pueden entrar en conflicto con un editor que rechace sistemáticamente la mayor parte de las aportaciones. Estas situaciones existen realmente en Wikipedia y, normalmente, se solucionan en un plazo de tiempo medio, como es propio de los proyectos abiertos y meritocráticos. Pero este plazo de tiempo puede resultar demasiado largo para el horizonte temporal en que se haya planteado el trabajo. Por ello, previamente al inicio del trabajo, el profesor deberá explicar y guiar a los alumnos por los usos y costumbres básicos de la edición de Wikipedia, con especial atención a la gestión de los conflictos.

En este contexto resulta de especial interés el uso de las páginas de discusión e historial acerca de cada entrada. El profesor deberá instruir a los alumnos en 
como a través de ellas pueden mantener un debate con otros editores acerca de la conveniencia de sus aportaciones. Además, este debate contribuiría a desarrollar sus capacidades de razonamiento crítico por lo que debería verse como una aportación adicional a la capacidad formadora del trabajo escrito. En última instancia, la página de historial reflejará toda su actividad, por lo que la capacidad de ser evaluados no se verá disminuida aunque otros editores revoquen sus cambios.

Entre los resultados esperados que se derivarían de este cambio en la forma de realizar los trabajos escritos destacamos:

- Mejora de la motivación ante los trabajos, tanto en el plano de los contenidos como en el de la presentación (estructuración, redacción, relevancia...).

- Paso de rol del alumno de usuario a creador de información original.

- $\quad$ Reforzamiento de las habilidades de consulta y cita de fuentes.

- Aprender a trabajar en entornos colaborativos.

- Fomento del conocimiento abierto como un valor intrínseco al proceso educativo.

- Espíritu de mejora continua transfiriendo los resultados a cursos futuros, evitando que cada trabajo de la asignatura sea un esfuerzo aislado.

No debemos cerrar este apartado sin destacar que muchas herramientas de campus virtual, como pueden ser Moodle, WebCT o Sakai, permiten la realización de wikis dentro de su entorno de aprendizaje colaborativo. No obstante, creemos que el trabajo con una herramienta como Wikipedia resulta más motivador y provechoso por una doble razón. En primer lugar, el trabajo sobre una obra pública como Wikipedia debe aumentar la motivación de los alumnos ante su exposición y posteriores revisiones por terceros ajenos a la asignatura. En segundo lugar, los entornos de campus virtual suponen la adquisición de habilidades en el manejo de herramientas solo empleadas en instituciones educativas; por el contrario, el empleo de herramientas abiertas, como es el caso de Wikipedia y su entorno de software MediaWiki, permite la adquisición de habilidades que en el futuro puedan servir a un propósito más amplio. 


\section{CONCLUSIÓN}

Internet es actualmente la principal fuente de búsqueda de información en la realización de trabajos escritos, destacando el uso de Google y Wikipedia. Muchos alumnos realizan copia y pega de la información encontrada para formar sus trabajos sin realizar ningún tipo de atribución a las fuentes. Creemos conveniente desarrollar estrategias que encaucen estos comportamientos en favor de la mejora y la calidad en vez de simplemente penalizarlos cuando son descubiertos.

El uso de Wikipedia para el desarrollo de trabajos escritos debería suponer un proceso de mejora continua en el proceso de enseñanza-aprendizaje a través de los sucesivos semestres. Además, introduciría al alumno en la filosofía del conocimiento libre y colaborativo, dándole a conocer su creciente importancia en la cultura digital y la trascendencia de un rol activo en ella como creadores. Por último, el estímulo de una audiencia global debería beneficiar el rigor en la realización del trabajo, así como el estilo de redacción y riqueza gramatical.

Los cambios en la educación deben correr paralelos a los cambios sociales. Las actuales generaciones de estudiantes han crecido en un entorno en el que ciertos materiales como los libros impresos les pueden resultar en gran parte anacrónicos. Acostumbrados al manejo de la información libremente gracias a estándares abiertos y relacionales como los que han impulsado el crecimiento de la Web, resulta cuando menos absurdo obligarles a ceñirse a lo que pueden percibir como materiales, usos y costumbres obsoletos y que resultan menos eficientes en la transmisión de conocimiento.

Se hace necesario vencer la resistencia que parte de los docentes presentan ante el uso de fuentes no impresas y, en particular, ante el uso de Wikipedia como herramienta de aprendizaje. Máxime cuando nos encontramos ante un recurso de elevado valor para los procesos de aprendizaje a distancia, tanto en su vertiente económica como en su vertiente académica. No obstante, no se ha de plantear este cambio como una simple transformación tecnológica. Si la tecnología digital ofrece un vehículo más rápido y más eficiente de transmisión del conocimiento, es misión de los profesores orientar al alumno en el uso de dicho vehículo y velar para que al mismo tiempo que se adquieren conocimientos, se desarrollen las suficientes habilidades críticas en el tratamiento de la información que eviten las creencias de que todo aquello que está en Internet es cierto, del mismo modo que no todo aquello que estaba impreso era cierto en el pasado. 


\section{REFERENCIASBIBLIOGRÁFICAS}

Alexandersson, M.; Limberg, L. (2003). Constructing meaning through information artefacts. New Review of Information Behaviour Research, 4 (1), (17-30).

Budapest Open Access Initiative (2001). Mission statement. [en línea] Disponible en: http://www.soros.org/ openaccess/read (consulta 2011, 4 de diciembre).

Declaración de Berlín sobre Acceso Abierto (2003). Texto de la declaración. [en línea] Disponible en: http://www.zim. mpg.de/openaccess-berlin/berlin declaration.pdf (consulta 2011, 4 de diciembre).

Forte, A.; Bruckman, A. (2010). Writing, citing, and participatory media: wikis as learning environments in the high school classroom. International Journal of Learning and Media, 1 (4), (23-44).

Free Software Foundation (1986). Definición de Software Libre. [en línea] Disponible en: http://www.gnu.org/philosophy/ free-sw.es.html (consulta 2011, 4 de diciembre).

Giles, J. (2005). Internet encyclopaedias go head to head. Nature, 438, (900-901).

Gourdain, P.; O'Kelly, P.; Roman-Amat, B.; Soulas, D.; von Droste zu Hülshoff, T. (2008). La revolución Wikipedia. Madrid: Alianza Editorial.

Head, A. J.; Eisenberg, M. B. (2010). How today's college students use Wikipedia for course-related research. First Monday, 15 (3-1) [en línea] Disponible en: $\quad$ http://www.uic.edu/htbin/ cgiwrap/bin/ojs/index.php/fm/article/ view/2830/24766 (consulta 2011, 4 de diciembre).

Heinström, J. (2006). Fast surfing for availability or deep diving into quality, motivation and information seeking among middle and high school students. Information Research, 11 (4).
Konieczny, P. (2010). Teaching with Wikipedia and other Wikimedia Foundation wikis. 6th International Simposium on Wikis and Open Collaboration WikiSym, Gdansk, Polonia.

Limberg, L.; Alexandersson, M.; LantzAndersson, A.; Folkesson, L. (2008). What matters? Shaping meaningful learning through teaching information literacy. Libri, 58 (2), (82-91).

Open Knowledge Foundation (2004). Definición de conocimiento abierto, versión 1.1. [en línea] Disponible en: http://opendefinition.org/okd/ (consulta 2011, 4 de diciembre).

Sánchez Vera, M. M. (2012). Diseño de recursos digitales para entornos de e-learning en la enseñanza universitaria. RIED. Revista Iberoamericana de Educación a Distancia, 15 (2), (53-74).

Schulenburg, F.; Wannemacher, K. (2009). Wikipedia in Academic Studies. Corrupting or Dramatically Improving the Quality of Teaching and Learning? En: Ebner, P.; Schiefner, M. (eds.). Looking Toward the Future of Technology Enhanced Education: Ubiquitous Learning and the Digital Native. Pasadena: Hershey.

Sormunen, E.; Lehtiö, L. (2011). Authoring Wikipedia articles as an information literacy assignment: copy-pasting or expressing new understanding in one's own words? Information Research, 16 (4).

Wikipedia (2009). Licencia de contenido. [en línea] Disponible en: $\quad$ https://es.wikipedia.org/wiki/ Wikipedia\#Licencia_de_contenido (consulta 2011, 13 de diciembre).

Wikipedia (2011). Wikipedia in Education. [en línea] Disponible en: http:// outreach.wikimedia.org/wiki/ Education (consulta 2011, 13 de diciembre). 


\section{PERFIL ACADÉMICO Y PROFESIONAL DE LOS AUTORES}

María Isabel Alonso de Magdaleno. Profesora titular en el Departamento de Administración de Empresas de la Universidad de Oviedo. Doctora en Dirección y Administración de Empresas por la Universidad de Oviedo. Su actividad investigadora se ha centrado en la dinámica de sistemas aplicada a la gestión sanitaria y a los procesos colaborativos y participativos a través de la Red.

E-mail: ialonso@uniovi.es

Jesús García García. Profesor contratado doctor en el Departamento de Contabilidad de la Universidad de Oviedo. Doctor en Economía Financiera y Contabilidad por la Universidad de Oviedo. Su campo de investigación se centra en el estudio del impacto económico y social de los fenómenos de transparencia, apertura y colaboración en la Red.

E-mail: jesgar@uniovi.es

DIRECCIÓN DE LOS AUTORES

Facultad de Economía y Empresa

Avenida del Cristo s/n.

33071 Oviedo (España)

Fecha de recepción del artículo: 15/05/2012

Fecha de aceptación del artículo: 16/09/2012

\section{Como citar este artículo:}

Alonso de Magdaleno, M. I.; García García, J. (2013). Colaboración activa en wikipedia como método de aprendizaje. RIED. Revista Iberoamericana de Educación a Distancia, volumen 16, nº 1, pp. 13-26. 